\title{
On the General Hermite Cardinal Interpolation
}

\author{
By R. Kress
}

\begin{abstract}
A sequence of interpolation series is given which generalizes Whittaker's cardinal function to the case of Hermite interpolation. By integrating the interpolation series, a sequence of new quadrature formulae for $\int_{-\infty}^{\infty} f(x) d x$ is obtained. Derivative-free remainders are stated for these interpolation and quadrature formulae.
\end{abstract}

Given a function $f: \mathbf{R} \rightarrow \mathbf{C}$ and a real number $h>0$, the series

$$
\begin{aligned}
T_{h}(f)(z): & =\frac{h}{\pi} \sum_{m=-\infty}^{\infty} \frac{(-1)^{m} f(m h)}{z-m h} \sin \frac{\pi}{h} z \\
& =\frac{h}{\pi} \sum_{m=-\infty}^{\infty} \frac{f(m h)}{z-m h} \sin \frac{\pi}{h}(z-m h), \quad z \in \mathbf{C},
\end{aligned}
$$

is called the cardinal series of the function $f$ with respect to the interval $h$. If the series converges, its sum $T_{h}(f)$ is called the cardinal function or cardinal interpolation of the function $f$. Obviously,

$$
T_{h}(f)(m h)=f(m h), \quad m=0, \pm 1, \pm 2, \cdots,
$$

holds. In the case when $f: B \rightarrow \mathbf{C}$ is analytic in a strip $B:=\mathbf{R} \times[-a, a] \subset \mathbf{C}, a>0$, and satisfies certain conditions at infinity, a derivative-free remainder for this cardinal interpolation was independently found by Kress [2] and McNamee, Stenger and Whitney [6].

In the present paper, we generalize the cardinal interpolation and give a sequence of Hermite cardinal interpolations $T_{p, h}(f), p=0,1,2, \cdots$, with

$$
T_{p, h}^{(q)}(f)(m h)=f^{(a)}(m h), \quad q=0,1, \cdots, p, \quad m=0, \pm 1, \pm 2, \cdots .
$$

The usual cardinal interpolation is included as the particular case $p=0$.

In Section 1, we give the explicit form of $T_{p, h}(f)$ and state a derivative-free remainder. Making use of this remainder, we describe a class of functions for which $T_{p, h}(f)=f$.

In Section 2, we apply the general cardinal functions to derive a sequence $I_{p, h}(f)$, $p=0,2,4, \cdots$, of integration formulae for infinite integrals involving the derivatives $f^{(q)}(m h), q=0,2, \cdots, p, m=0, \pm 1, \pm 2, \cdots$, which may be regarded as generalizations of the trapezoidal rule. The remainder, given by Goodwin [1], Martensen [4] and McNamee [5] for the trapezoidal rule, is extended to the quadrature formulae $I_{p, h}(f)$.

Received December 27, 1971.

AMS 1970 subject classifications. Primary 30A80, 41A05, 41A55.

Key words and phrases. Cardinal function, Hermite interpolation, quadrature formulae, analytic functions, remainders, error bounds.

Copyright $\odot 1972$, American Mathematical Society 
The general cardinal interpolation developed in this paper is closely related to the general Hermite trigonometric interpolation of periodic functions [3].

1. Interpolation. Let $p \geqq 0$ be an integer and let $h>0$ be real. Define $p+1$ entire functions $t_{a}, q=0,1, \cdots, p$, by

$$
t_{a}(z):=\frac{z^{a}}{q !}\left(\frac{\sin (\pi / h) z}{(\pi / h) z}\right)^{p+1} \sum_{r=0 ; r \text { even }}^{2[(p-q) / 2]} a_{r}(p)\left(\frac{\pi}{h} z\right)^{r}, \quad z \in \mathbf{C},
$$

where the $a_{r}(p)$ are the coefficients of the Laurent expansion

$$
\frac{1}{\sin ^{p+1} z}=\sum_{r=0 ; r \text { even }}^{\infty} \frac{a_{r}(p)}{z^{1+p-r}}, \quad 0<|z|<\pi .
$$

To avoid indexing difficulties, we do not indicate the dependence of the $t_{q}$ on $p$ and $h$.

LEMMA 1.1. For every $r=0,1, \cdots$, the functions $t_{q}, q=0,1, \cdots$, satisfy

$$
\begin{aligned}
t_{q}^{(r)}(0) & =\delta_{q}^{r}, \\
t_{q}^{(r)}(m h) & =0, \quad m= \pm 1, \pm 2, \cdots .
\end{aligned}
$$

Proof. From (1.1) and (1.2), we obtain

$$
t_{q}(z)=z^{q} / q !+z^{p+1} u_{q}(z), \quad q=0,1, \cdots, p,
$$

with certain entire functions $u_{q}$, and (1.3) immediately follows. The relation (1.4) trivially holds.

Definition 1.1. Let $p \geqq 0$ be an integer and let $h>0$ be real. Given a function $f: \mathbf{R} \rightarrow \mathbf{C}, f \in C^{p}(\mathbf{R})$, the pth cardinal series of $f$ with respect to the interval $h$ is defined by

$$
T_{p, h}(f)(z):=\sum_{m=-\infty}^{\infty} \sum_{q=0}^{p} f^{(a)}(m h) t_{a}(z-m h), \quad z \in \mathbf{C} .
$$

If the series converges, its sum $T_{p, h}(f)$ is called the pth cardinal function of $f$.

Lemma 1.1 implies

THEOREM 1.1. The pth cardinal function $T_{p, h}(f)$ is a Hermite interpolation of the function $f$ with equidistant interpolation points

$$
T_{p, h}^{(q)}(f)(m h)=f^{(q)}(m h), \quad q=0,1, \cdots, p, \quad m=0, \pm 1, \cdots .
$$

The first cardinal series is listed below.

$$
\begin{aligned}
& T_{0, h}(f)(z)= \frac{h}{\pi} \sum_{m=-\infty}^{\infty}(-1)^{m} \frac{f(m h)}{z-m h} \sin \frac{\pi}{h} z, \\
& T_{1, h}(f)(z)=\left(\frac{h}{\pi}\right)^{2} \sum_{m=-\infty}^{\infty}\left\{\frac{f(m h)}{(z-m h)^{2}}+\frac{f^{\prime}(m h)}{z-m h}\right\} \sin ^{2} \frac{\pi}{h} z, \\
& T_{2, h}(f)(z)=\left(\frac{h}{\pi}\right)^{3} \sum_{m=-\infty}^{\infty}(-1)^{m}\left\{\frac{f(m h)}{(z-m h)^{3}}-\frac{1}{2}\left(\frac{\pi}{h}\right)^{2} \frac{f(m h)}{z-m h}\right. \\
&\left.\quad+\frac{f^{\prime}(m h)}{(z-m h)^{2}}+\frac{f^{\prime \prime}(m h)}{z-m h}\right\} \sin ^{3} \frac{\pi}{h} z .
\end{aligned}
$$

In the case when the function $f$ is analytic in a strip $B:=\mathbf{R} \times[-a, a] \subset \mathbf{C}$, $a>0$, we shall give a sufficient condition on the convergence of the $p$ th cardinal 
series of $f$ and shall obtain a representation of the remainder

$$
R_{p, h}(f):=f-T_{p, h}(f) .
$$

LEMMA 1.2. Let the function $f$ be analytic in the strip $B:=\mathbf{R} \times[-a, a] \subset \mathbf{C}$, $a>0$. Then

$$
\begin{aligned}
\chi_{n}(z) f(z)-\sum_{m=-n}^{n} & \sum_{a=0}^{p} f^{(a)}(m h) t_{q}(z-m h) \\
= & \frac{1}{2 \pi i} \sin ^{p+1} \frac{\pi}{h} z \int_{C_{n}} \frac{f(\zeta) d \zeta}{(\zeta-z) \sin ^{p+1}(\pi / h) \zeta}, \quad z \notin C_{n},
\end{aligned}
$$

where $C_{n}$ denotes the boundary of a rectangle $B_{n}:=\left[-\left(n+\frac{1}{2}\right) h,\left(n+\frac{1}{2}\right) h\right] \times$ $[-a, a] \subset B$ and where $\chi_{n}$ denotes the characteristic function of $B_{n}$ with $\chi_{n}(z)=1$, $z \in B_{n}$ and $\chi_{n}(z)=0, z \notin B_{n}$.

Proof. The function $F: B_{n} \rightarrow \mathrm{C}$, defined by

$$
F(z):=\frac{1}{\sin ^{p+1}(\pi / h) z}\left(f(z)-\sum_{m=-n}^{n} \sum_{q=0}^{p} f^{(q)}(m h) t_{q}(z-m h)\right), \quad z \in B_{n},
$$

is analytic. Hence, by Cauchy's theorem,

$$
\chi_{n}(z) F(z)=\frac{1}{2 \pi i} \int_{C_{n}} \frac{F(\zeta)}{\zeta \subsetneq z} d \zeta, \quad z \notin C_{n} .
$$

Using the identities

$$
\int_{C_{n}} \frac{d \zeta}{(\zeta-z)(\zeta-m h)^{a+1}}=\frac{-2 \pi i}{(z-m h)^{\alpha+1}}\left(1-\chi_{n}(z)\right), \quad z \notin C_{n},
$$

$q=0,1, \cdots, p, m=0, \pm 1, \cdots, \pm n$, we substitute (1.9) into (1.10) and obtain (1.8).

THEOREM 1.2. Let the function $f$ be analytic and bounded in the strip $B:=\mathbf{R} \times$ $[-a, a] \subset \mathrm{C}, a>0$, and let

$$
\int_{-\infty-i a}^{\infty-i a}|f(z)|^{2} d s<\infty, \quad \int_{-\infty+i a}^{\infty+i a}|f(z)|^{2} d s<\infty .
$$

Then, for arbitrary $p \geqq 0$ and $h>0$, the pth cardinal series of $f$ with respect to the interval $h$ is locally uniformly convergent for all $x \in \mathbf{R}$ and the remainder (1.7) is given by

$$
\begin{aligned}
R_{p, h}(f)(x)=\frac{1}{2 \pi i} \sin ^{p+1} \frac{\pi}{h} x\{ & \int_{-\infty-i a}^{\infty-i a} \frac{f(\zeta) d \zeta}{(\zeta-x) \sin ^{p+1}(\pi / h) \zeta} \\
& \left.\quad-\int_{-\infty+i a}^{\infty+i a} \frac{f(\zeta) d \zeta}{(\zeta-x) \sin ^{p+1}(\pi / h) \zeta}\right\}, \quad x \in \mathbf{R},
\end{aligned}
$$

and bounded by

$$
\begin{aligned}
\left|R_{p, h}(f)(x)\right| \leqq \frac{1}{2(\pi a)^{1 / 2}}\left(\frac{\sin (\pi / h) x}{\sinh (\pi / h) a}\right)^{p+1}\{ & \left(\int_{-\infty-i a}^{\infty-i a}|f(\zeta)|^{2} d s\right)^{1 / 2} \\
& \left.+\left(\int_{-\infty+i a}^{\infty+i a}|f(\zeta)|^{2} d s\right)^{1 / 2}\right\}, \quad x \in \mathbf{R} .
\end{aligned}
$$


Proof. Let $f$ be bounded by $M$. Then we estimate the integrals

$$
\left|\int_{ \pm(n+1 / 2) h-i a}^{ \pm(n+1 / 2) h+i a} \frac{f(\zeta) d \zeta}{(\zeta-x) \sin ^{p+1}(\pi / h) \zeta}\right| \leqq \frac{2 M a}{\left(n+\frac{1}{2}\right) h-|x|}
$$

Thus, by Lemma 1.2,

$$
\begin{aligned}
& f(x)=\lim _{n \rightarrow \infty}\left[\sum_{m=-n}^{n} f^{(a)}(m h) t_{q}(x-m h)\right. \\
& +\frac{1}{2 \pi i} \sin ^{p+1} \frac{\pi}{h} x\left\{\int_{-(n+1 / 2) h-i a}^{+(n+1 / 2) h-i a} \frac{f(\zeta) d \zeta}{(\zeta-x) \sin ^{p+1}(\pi / h) \zeta}\right. \\
& \left.\left.\quad-\int_{-(n+1 / 2) h+i a}^{+(n+1 / 2) h+i a} \frac{f(\zeta) d \zeta}{(\zeta-x) \sin ^{p+1}(\pi / h) \zeta}\right\}\right], \quad x \in \mathbf{R},
\end{aligned}
$$

where convergence is locally uniform for all $x \in \mathbf{R}$. Upon noting that

$$
\left|\sin \frac{\pi}{h} \zeta\right| \geqq \sinh \frac{\pi}{h} a, \quad \zeta=\xi \pm i a,
$$

and

$$
\int_{-\infty \pm i a}^{\infty \pm i a} d s /|\zeta-x|^{2}=\pi / a
$$

Schwarz's inequality yields

$\left|\int_{-\infty \pm i a}^{\infty \pm i a} \frac{f(\zeta) d \zeta}{(\zeta-x) \sin ^{p+1}(\pi / h) \zeta}\right| \leqq \frac{\sqrt{ } \pi}{\sqrt{ } a \sinh ^{p+1}(\pi / h) a}\left(\int_{-\infty \pm i a}^{\infty \pm i a}|f(\zeta)|^{2} d s\right)^{1 / 2}, \quad x \in \mathbf{R}$.

Hence, letting $n \rightarrow \infty$ in (1.14) completes the proof.

Remark. From the bound (1.13), we easily see that

$$
\lim _{h \rightarrow 0} T_{p, h}(f)(x)=f(x), \quad p=0,1, \cdots,
$$

and

$$
\lim _{p \rightarrow \infty} T_{p, h}(f)(x)=f(x), \quad h>0, \quad \sinh \frac{\pi}{h} a>1,
$$

where convergence is uniform for all $x \in \mathrm{R}$. In both cases $h \rightarrow 0, p$ fixed and $p \rightarrow \infty$, $h$ fixed, the convergence is exponential.

The following theorem describes a class of functions for which $T_{p, h}(f)=f$ is true.

THEOREM 1.3. Let $f$ be an entire function, such that

$$
|f(z)| \leqq c e^{\rho|y|}, \quad z=x+i y \in \mathbf{c},
$$

with real numbers $c \geqq 0$ and $0 \leqq \rho<(p+1) \pi / h$. Then the pth cardinal series for $f$ with respect to $h$ is locally uniformly convergent for all $z \in \mathbf{C}$, and the identity

$$
T_{p, h}(f)(z)=f(z), \quad z \in \mathbf{C},
$$

holds.

Proof. By (1.15) we have 


$$
\begin{aligned}
\left|\frac{f(\zeta)}{\sin ^{p+1}(\pi / h) \zeta}\right| & \leqq \frac{c e^{\rho|\eta|}}{\sinh ^{p+1}(\pi / h)|\eta|} \\
& =o\left(\exp \left[-\left[(p+1) \frac{\pi}{h}-\rho\right]|\eta|\right]\right), \quad \zeta=\xi+i \eta \in \mathbf{c},
\end{aligned}
$$

as $|\eta| \rightarrow \infty$, and therefore

$$
\lim _{a \rightarrow \infty} \int_{-(n+1 / 2) h \pm i a}^{(n+1 / 2) h \pm i a} \frac{f(\zeta) d \zeta}{(\zeta-x) \sin ^{p+1}(\pi / h) \zeta}=0,
$$

that is, by Lemma 1.2,

$$
\begin{aligned}
& f(z)= \sum_{m=-n}^{n} \sum_{a=0}^{p} f^{(a)}(m h) t_{q}(z-m h) \\
&+\frac{1}{2 \pi i} \sin ^{p+1} \frac{\pi}{h} z\left\{\int_{(n+1 / 2) h-i \infty}^{(n+1 / 2) h+i \infty} \frac{f(\zeta)}{(\zeta-z) \sin ^{p+1}(\pi / h) \zeta} d \zeta\right. \\
& \\
&\left.\quad-\int_{-(n+1 / 2) h-i \infty}^{-(n+1 / 2) h+i \infty} \frac{f(\zeta)}{(\zeta-z) \sin ^{p+1}(\pi / h) \zeta} d \zeta\right\}, \\
& z=x+i y \in \mathbf{C}
\end{aligned}
$$

for all $n$ with $\left(n+\frac{1}{2}\right) h>|x|$. Making use of

$$
\left|\sin \frac{\pi}{h} \zeta\right|=\cosh \frac{\pi}{h} \eta \geqq \frac{1}{2} \exp \left[\frac{\pi}{h}|\eta|\right], \quad \zeta= \pm\left(n+\frac{1}{2}\right) h+i \eta,
$$

we conclude that

$$
\left|\int_{ \pm(n+1 / 2) h-i \infty}^{ \pm(n+1 / 2) h+i \infty} \frac{f(\zeta) d \zeta}{(\zeta-z) \sin ^{p+1}(\pi / h) \zeta}\right| \leqq \frac{2^{p+2} c}{(p+1)(\pi / h)-\rho} \frac{1}{\left(n+\frac{1}{2}\right) h-|x|} .
$$

Letting $n \rightarrow \infty$ in (1.17), the assertion of the theorem follows.

Example. If we choose $f(z):=e^{i \rho z}, z \in \mathrm{C}, 0 \leqq \rho<(p+1) \pi / h$, we obtain the local uniform convergent expansion

$$
e^{i \rho z}=\sum_{m=-\infty}^{\infty} \sum_{a=0}^{p}(i \rho)^{a} e^{i \rho m h} t_{q}(z-m h), \quad z \in \mathbf{C} .
$$

Setting $\rho:=r \pi / h, r=0,1, \cdots, p$, we derive

$$
\exp \left[i r \frac{\pi}{h} z\right]=\sum_{m=-\infty}^{\infty} \sum_{q=0}^{p}\left(i r \frac{\pi}{h}\right)^{Q}(-1)^{r m} t_{q}(z-m h), \quad z \in \mathrm{C} .
$$

2. Numerical Integration. We integrate the $p$ th cardinal series of $f$ termwise and obtain the series

$$
I_{p, h}(f):=h \sum_{m=-\infty}^{\infty} \sum_{q=0 ; q \text { even }}^{p}\left(\frac{h}{2 \pi}\right)^{q} a_{q, p} f^{(a)}(m h)
$$

with the weights

$$
a_{a, p}:=\frac{1}{2 \pi}\left(\frac{2 \pi}{h}\right)^{a+1} \int_{-\infty}^{\infty} t_{q}(x) d x, \quad q=0,2, \cdots, p .
$$


If $q$ is odd, the integral (2.2) vanishes, since in this case the function $t_{a}$ is odd. The series (2.1) may be regarded as generalizations of the trapezoidal rule approximation for the integral $\int_{-\infty}^{\infty} f(x) d x$.

In order to derive simple recurrence formulae for the weights $a_{a, p}$, we state

THEOREM 2.1. Let $p$ be even. Then the weights $a_{a, p}$ are uniquely determined by the identity

$$
\sum_{a=0 ; \propto \text { even }}^{p} a_{q, p^{\alpha}} z^{a}=\prod_{q=1}^{p / 2}\left(1+(z / q)^{2}\right),{ }^{*} \quad z \in \mathbf{C} .
$$

Proof. Integrating (1.19), we have

$$
\int_{0}^{h} \exp \left[\operatorname{ir} \frac{\pi}{h} x\right] d x=\sum_{a=0 ; q \text { even }}^{p}\left(\operatorname{ir} \frac{\pi}{h}\right)^{q} \int_{-\infty}^{\infty} t_{a}(x) d x, \quad r=0,2, \cdots, p,
$$

thus, we are led to the system of $p / 2+1$ linear equations

$$
a_{0, p}=1 \text {, }
$$

$$
\sum_{a=0 ; a \text { even }}^{p}(i r)^{a} a_{a, p}=0, \quad r=1, \cdots, p / 2 .
$$

Since the determinant $D_{p}$ of (2.4) is a Vandermonde determinant with

$$
D_{p}=i^{(p / 2)(p / 2+1)} \prod_{q>r=0}^{p / 2}\left(q^{2}-r^{2}\right) \neq 0,
$$

the weights $a_{a, p}$ are uniquely determined by the system (2.4).

Define a polynomial $P_{p}$ of degree $p$ by

$$
P_{p}(z):=\sum_{a=0 ; \varepsilon \text { even }}^{p} a_{a, p} z^{a}, \quad z \in \mathbf{C} .
$$

Then (2.4) reads

$$
\begin{aligned}
& P_{p}(0)=1, \\
& P_{p}(r i)=0, \quad r= \pm 1, \cdots, \pm p / 2 .
\end{aligned}
$$

Hence $P_{p}(z)=\prod_{q=1}^{p / 2}\left(1+(z / q)^{2}\right)$, and (2.3) is established.

From (2.3) it follows that

$$
\left(1+(2 z / p)^{2}\right) \sum_{q=0 ; q \text { even }}^{p-2} a_{a, p-2} z^{q}=\sum_{q=0 ; q \text { even }}^{p} a_{a, p} z^{q}, \quad p=2,4, \cdots .
$$

Comparing the coefficients, we find the desired recursion formulae

$$
\begin{aligned}
& a_{0, p}=1, \quad p=0,2, \cdots, \\
& a_{a, p}=a_{a, p-2}+(2 / p)^{2} a_{a-2, p-2}, \quad q=2,4, \cdots, p-2, \quad p=2,4, \cdots, \\
& a_{p, p}=\frac{1}{((p / 2) !)^{2}}, \quad p=0,2, \cdots .
\end{aligned}
$$

Using (2.5), we obtain

\footnotetext{
$* \Pi_{q=1}^{p / 2}$ is to be interpreted as unity when $p=0$.
} 


$$
\begin{aligned}
& I_{0, h}(f)=h \sum_{m=-\infty}^{\infty} f(m h), \\
& I_{2, h}(f)=h \sum_{m=-\infty}^{\infty} f(m h)+\frac{h^{3}}{4 \pi^{2}} \sum_{m=-\infty}^{\infty} f^{\prime \prime}(m h), \\
& I_{4, h}(f)=h \sum_{m=-\infty}^{\infty} f(m h)+\frac{5 h^{3}}{16 \pi^{2}} \sum_{m=-\infty}^{\infty} f^{\prime \prime}(m h)+\frac{h^{5}}{64 \pi^{4}} \sum_{m=-\infty}^{\infty} f^{\prime \prime \prime \prime}(m h) .
\end{aligned}
$$

In the case of odd $p$, we integrate (1.19) for $r=0,2, \cdots, p-1$ and get the system of $(p+1) / 2$ linear equations

$$
\begin{aligned}
a_{0, p} & =1, \\
\sum_{a=0 ; \alpha \text { even }}^{p-1}(\text { ir })^{a} a_{a, p} & =0, \quad r=1, \cdots,(p-1) / 2 .
\end{aligned}
$$

Comparing (2.6) with (2.4), we see that $a_{q, p}=a_{q, p-1}, q=0,2, \cdots, p-1$. Thus, if $p$ is odd, $I_{p, h}(f)=I_{p-1, h}(f)$ is valid. Therefore, we may restrict ourselves to even $p$.

In the case when the function $f$ is analytic, we state a sufficient condition on the convergence of the series (2.1) and give a remainder in the following:

THEOREM 2.2. Let the function $f$ be analytic in the strip $B:=\mathrm{R} \times[-a, a] \subset \mathbf{C}$, $a>0$, let $f(z) \rightarrow 0, z=x+$ iy as $x \rightarrow \pm \infty$ uniformly for all $-a \leqq y \leqq a$ and let

$$
\int_{-\infty-i a}^{\infty-i a}|f(z)| d s<\infty, \quad \int_{-\infty+i a}^{\infty+i a}|f(z)| d s<\infty .
$$

Then $\int_{-\infty}^{\infty} f(x) d x$ exists, and the series (2.1) is convergent for even $p \geqq 0$ and $h>0$. The remainder

$$
E_{p, h}(f):=\int_{-\infty}^{\infty} f(x) d x-I_{p, h}(f)
$$

is given by

$$
E_{p, h}(f)
$$

$$
\begin{aligned}
=\frac{1}{(2 i)^{p+1}} \sum_{a=0}^{p / 2}(-1)^{q}\left(\begin{array}{c}
p+1 \\
q
\end{array}\right) & \left\{\int_{-\infty+i a}^{\infty+i a} \frac{\exp [i(p+1-2 q)(\pi / h) \zeta]}{\sin ^{p+1}(\pi / h) \zeta} f(\zeta) d \zeta\right. \\
& \left.-\int_{-\infty-i a}^{\infty-i a} \frac{\exp [-i(p+1-2 q)(\pi / h) \zeta]}{\sin ^{p+1}(\pi / h) \zeta} f(\zeta) d \zeta\right\}
\end{aligned}
$$

with the bound

$$
\left|E_{p, h}(f)\right| \leqq \frac{\exp [-(\pi / h) a]}{2 \sinh ^{p+1}(\pi / h) a}\left(\int_{-\infty+i a}^{\infty+i a}|f(z)| d s+\int_{-\infty-i a}^{\infty-i a}|f(z)| d s\right) .
$$

Proof. From the assumption (2.7), we see by Cauchy's theorem that $\int_{-\infty}^{\infty} f(x) d x$ exists.

Using the identity

$$
\sin ^{p+1} \frac{\pi}{h} x=\frac{\exp [i(p+1)(\pi / h) x]}{(2 i)^{p+1}} \sum_{q=0}^{p+1}(-1)^{q}\left(\begin{array}{c}
p+1 \\
q
\end{array}\right) \exp [-2 i q(\pi / h) x],
$$


we deduce from the residue theorem that

$\frac{1}{\pi} \int_{-\infty}^{\infty} \frac{\sin ^{p+1}(\pi / h) x}{x-\zeta} d x=\frac{\exp [i(p+1)(\pi / h) \zeta]}{(2 i)^{p}} \sum_{q=0}^{p / 2}(-1)^{q}\left(\begin{array}{c}p+1 \\ q\end{array}\right) \exp [-2 i q(\pi / h) \zeta]$, $\zeta=\xi+i a$,

$\frac{1}{\pi} \int_{-\infty}^{\infty} \frac{\sin ^{p+1}(\pi / h) x}{x-\zeta} d x=\frac{\exp [-i(p+1)(\pi / h) \zeta]}{(2 i)^{p}} \sum_{q=0}^{p / 2}(-1)^{q}\left(\begin{array}{c}p+1 \\ q\end{array}\right) \exp [2 i q(\pi / h) \zeta]$,

$$
\zeta=\xi-i a
$$

From this the estimates

$$
\begin{aligned}
\left|\frac{1}{\pi} \int_{-\infty}^{\infty} \frac{\sin ^{p+1}(\pi / h) x}{x-\zeta} d x\right| & \leqq \frac{\exp [-(p+1)(\pi / h) a]}{2^{p}} \sum_{a=0}^{p / 2}\left(\begin{array}{c}
p+1 \\
q
\end{array}\right) \exp [2 q(\pi / h) a] \\
& \leqq \exp [-(\pi / h) a], \quad \zeta=\xi \pm i a,
\end{aligned}
$$

follow.

Integrating (1.8) over $(-\infty, \infty)$ and interchanging the order of integration, we obtain

$$
\begin{aligned}
\int_{-(n+1 / 2) h}^{(n+1 / 2) h} f(x) d x= & h \sum_{m=-n}^{n} \sum_{a=0}^{p}\left(\frac{h}{2 \pi}\right)^{a} a_{a, p} f^{(a)}(m h) \\
& +\frac{1}{2 \pi i} \int_{C_{n}} \frac{f(\zeta)}{\sin ^{p+1}(\pi / h) \zeta}\left(\int_{-\infty}^{\infty} \frac{\sin ^{p+1}(\pi / h) x}{\zeta-x} d x\right) d \zeta .
\end{aligned}
$$

With the aid of (2.11), we can estimate

$$
\begin{array}{r}
\left|\frac{1}{\pi} \int_{ \pm(n+1 / 2) h-i a}^{ \pm(n+1 / 2) h+i a} \frac{f(\zeta)}{\sin ^{p+1}(\pi / h) \zeta}\left(\int_{-\infty}^{\infty} \frac{\sin ^{p+1}(\pi / h) x}{\zeta-x} d x\right) d \zeta\right| \\
\leqq 2 a \max _{\eta \in[-a, a]}\left|f\left( \pm\left(n+\frac{1}{2}\right) h+i \eta\right)\right|,
\end{array}
$$

and

$$
\begin{aligned}
\left|\frac{1}{\pi} \int_{-(n+1 / 2) h \pm i a}^{(n+1 / 2) h \pm i a} \frac{f(\zeta)}{\sin ^{p+1}(\pi / h) \zeta}\left(\int_{-\infty}^{\infty} \frac{\sin ^{p+1}(\pi / h) x}{\zeta-x} d x\right) d \zeta\right| \\
\leqq \frac{\exp [-(\pi / h) a]}{\sinh ^{p+1}(\pi / h) a} \int_{-(n+1 / 2) h \pm i a}^{(n+1 / 2) h \pm i a}|f(\zeta)| d s
\end{aligned}
$$

Thus, by the assumptions on $f$, letting $n \rightarrow \infty$ completes the proof.

Remark. From the bound (2.10), we have

$$
\lim _{h \rightarrow 0} I_{p, h}(f)=\int_{-\infty}^{\infty} f(x) d x, \quad p=0,2, \cdots,
$$

and

$$
\lim _{p \rightarrow \infty} I_{p, h}(f)=\int_{-\infty}^{\infty} f(x) d x, \quad h>0, \sinh \frac{\pi}{h} a>1,
$$

where convergence is exponential in both cases $h \rightarrow 0$, fixed and $p \rightarrow \infty, h$ fixed. 
Universität Göttingen

Institut für Numerische und Angewandte Mathematik

D-34, Göttingen, Germany

1. E. T. Goodwin, "The evaluation of integrals of the form $\int_{-x}^{\infty} f(x) \exp \left(-x^{2}\right) d x$," Proc. Cambridge Philos. Soc., v. 45, 1949, pp. 241-245. MR 10, 575.

2. R. KRESS, "Interpolation auf einem unendlichen Intervall," Computing, v. 6, 1971, pp. 274-288.

3. R. KRESS, "On the general Hermite trigonometric interpolation," Numer. Math. (To appear.)

4. E. Martensen, "Zur numerischen Auswertung uneigentlicher Integrale," Z. Angew. Math. Mech., v. 48, 1968, pp. T83-T85. MR 41 \#1221.

5. J. MCNAMEE, "Error-bounds for the evaluation of integrals by the Euler-MacLaurin formula and by Gauss-type formulae," Math. Comp., v. 18, 1964, pp. 368-381. MR 32 \#3264.

6. J. McNamee, F. Stenger \& E. L. Whitney, "Whittaker's cardinal function in retrospect," Math. Comp., v. 25, 1971, pp. 141-154. 